VOL. $61(2000)$ [387-403]

\title{
CHARACTERISTIC POLYNOMIALS OF FINITELY GENERATED MODULES OVER WEYL ALGEBRAS
}

\author{
Alexander B. Levin
}

\begin{abstract}
In this paper we modify the classical Gröbner basis technique and prove the existence of a characteristic polynomial in two variables associated with a finitely generated module over a Weyl algebra. We determine invariants of such a polynomial and show that some of the invariants are not carried by the Bernstein dimension polynomial of the module.
\end{abstract}

\section{INTRODUCTION}

The role of Hilbert polynomials in commutative algebra and algebraic geometry is well known. In [2] Bernstein introduced an analog of the Hilbert polynomial for a finitely generated filtered module over a Weyl algebra and extended the theory of multiplicity to the class of such modules. The results of this study have found interesting analytical applications (many of them are considered in Björk's book [4]). In particular, they allowed Bernstein [3] to prove the Gelfand's conjecture on meromorphic extensions of functions $\Gamma_{f}(\lambda)=\int P^{\lambda}(x) f(x) d x$ of one complex variable $\lambda$ defined in the half-space $R e(\lambda)>0$ for any polynomial in $n$ real variables $P(x)=P\left(x_{1}, \ldots, x_{n}\right)$ and for any function $f(x)=f\left(x_{1}, \ldots, x_{n}\right) \in C_{0}^{\infty}\left(\mathbf{R}^{n}\right)$.

In what follows we prove the existence, determine invariants and outline methods of computation of dimension polynomials in two variables associated with the natural bifiltration of a finitely generated module over a Weyl algebra $A_{n}(K)$. We show that such polynomials not only characterise the Bernstein class of left $A_{n}(K)$-modules, but also carry, in general, more invariants than the dimension polynomials introduced by Bernstein.

\section{Preliminaries}

Throughout the paper $\mathbf{Z}, \mathbf{N}$ and $\mathbf{Q}$ denote the sets of all integers, all non-negative integers and all rational numbers, respectively. As usual, $Q[t]$ denotes the ring of polynomials in one variable $t$ with rational coefficients and $o\left(t^{n}\right)$ denotes a polynomial from $Q[t]$ of degree less than $n$. By a ring we always mean an associative ring with a

Received 19th July, 1999

Copyright Clearance Centre, Inc. Serial-fee code: 0004-9727/00 \$A2.00+0.00. 
unit. Every ring homomorphism is unitary (maps unit onto unit), every subring of a ring contains the unit of the ring. Unless otherwise indicated, by a module over a ring $R$ we mean a unitary left $R$-module.

In what follows we consider a Weyl algebra as an algebra of differential operators over a polynomial ring. More precisely, let $K$ be a field of zero characteristic and $R=K\left[x_{1}, \ldots, x_{n}\right]$ a polynomial ring in $n$ variables $x_{1}, \ldots, x_{n}$ over $K$. Furthermore, let $\partial_{i}$ denote the operator of partial differentiation of the ring $R$ with respect to the variable $x_{i}(i=1, \ldots, n)$ and let $A_{n}(K)$ denote the corresponding ring of differential operators over $R$. Then $A_{n}(K)$ is said to be a Weyl algebra in $n$ variables with coefficients from $K$. It is clear that the $K$-algebra $A_{n}(K)$ is generated by the elements $x_{1}, \ldots, x_{n}, \partial_{1}, \ldots, \partial_{n}, \partial_{i} \partial_{j}=\partial_{j} \partial_{i}$ and $\partial_{i} x_{j}=x_{j} \partial_{i}$ for any two different indices $i$ and $j$ $\left(1 \leqslant i, j \leqslant n\right.$ ), and $\partial_{i} x_{i}=x_{i} \partial_{i}+1$ for $i=1, \ldots, n$. (The last identity is a consequence of the product rule, if one considers actions of the operators $\partial_{i} x_{i}$ and $x_{i} \partial_{i}$ on the ring $R:\left(\partial_{i} x_{i}\right)(P)=\partial_{i}\left(x_{i} P\right)=\left(x_{i} \partial_{i}\right)(P)+P$ for any $\left.P \in R.\right)$

In what follows, multi-indices with non-negative integers are denoted by small Greek letters. Thus, monomials $x_{1}^{\alpha_{1}} \ldots x_{n}^{\alpha_{n}}$ and $\partial_{1}^{\beta_{1}} \ldots \partial_{n}^{\beta_{n}}$ are written as $x^{\alpha}$ and $\partial^{\beta}$, their total degrees $\alpha_{1}+\cdots+\alpha_{n}$ and $\beta_{1}+\cdots+\beta_{n}$ are denoted by $|\alpha|$ and $|\beta|$, respectively.

It is known (see [4, Chapter 1 , Proposition 1.2]) that the monomials $x^{\alpha} \partial^{\beta}(\alpha, \beta \in$ $\mathrm{N}^{n}$ ) form a basis of $A_{n}(K)$ over the field $K$, so that every element $D \in A_{n}(K)$ can be written in a unique way as a finite $\operatorname{sum} \sum k_{\alpha \beta} x^{\alpha} \partial^{\beta}$ with the coefficients $k_{\alpha \beta} \in K$. The number ord $D=\max \left\{|\alpha|+|\beta| \mid k_{\alpha \beta} \neq 0\right\}$ is called the order of the element $D$.

Since $\operatorname{ord}\left(D_{1} D_{2}\right)=\operatorname{ord} D_{1}+\operatorname{ord} D_{2}$ for any $D_{1}, D_{2} \in A_{n}(K)$, the Weyl algebra $A_{n}(K)$ can be considered as a filtered ring with the nondecreasing filtration $\left(W_{r}\right)_{r \in Z}$ where $W_{r}=\left\{D \in A_{n}(K) \mid\right.$ ord $\left.D \leqslant r\right\}$ for $r \in \mathbf{N}$ and $W_{r}=0$, if $r<0$.

If $M$ is a finitely generated left $A_{n}(K)$-module with a system of generators $g_{1}, \ldots, g_{p}$, then $M$ can be naturally considered as a filtered $A_{n}(K)$-module with the filtration $\left(M_{r}\right)_{r \in Z}$ where $M_{r}=\sum_{i=1}^{p} W_{r} g_{i}$ for $r \in \mathbf{Z}$. It is clear that each $M_{r}$ is a finitely generated vector $K$-space, $W_{r} M_{s}=M_{r+s}$ for all $r, s \in \mathrm{N}$, and $\bigcup_{r \in \mathbb{N}} M_{r}=M$.

The following statement is proved in [2] (see also [4, Chapter 1, Corollaries 3.3, 3.5, and Theorem 4.1]).

PROPOSITION 2.1. With the above notation, there exists a polynomial $\psi_{M}(t) \epsilon$ $\mathrm{Q}[t]$ with the following properties.

(i) $\psi_{M}(r)=\operatorname{dim}_{K} M_{r}$ for all sufficiently large $r \in \mathbf{Z}$ (that is, there exists $r_{0} \in \mathbf{Z}$ such that the last equality holds for all integers $r \geqslant r_{0}$ );

(ii) $n \leqslant \operatorname{deg} \psi(t) \leqslant 2 n$; 
(iii) If $\psi(t)=a_{d} t^{d}+\cdots+a_{1} t+a_{0}\left(a_{d}, \ldots, a_{1}, a_{0} \in \mathbf{Q}\right)$, then the degree $d$ of the polynomial $\psi(t)$ and the integer $d ! a_{d}$ do not depend on the choice of the system of generators $g_{1}, \ldots, g_{p}$ of $M$. These numbers are denoted by $d(M)$ and $e(M)$; they are called the Bernstein dimension and multiplicity of the module $M$, respectively.

The polynomial $\psi_{M}(t)$ is called the Bernstein polynomial of the $A_{n}(K)$-module $M$ associated with the given system of generators. The family of all finitely generated left $A_{n}(K)$-modules $M$ such that $d(M)=n$ is denoted by $\mathcal{B}_{n}$; it is called the Bernstein class of $A_{n}(K)$-modules.

The following statement (see [4, Chapter 1, Proposition 5.2, 5.3 and Theorem 5.4]) gives some properties of the Bernstein class.

\section{Proposition 2.2.}

(i) If $0 \rightarrow M_{1} \rightarrow M_{2} \rightarrow M_{3} \rightarrow 0$ is an exact sequence of left $A_{n}(K)$-modules, then $M_{2} \in \mathcal{B}_{n}$ if and only if $M_{1} \in \mathcal{B}_{n}$ and $M_{3} \in \mathcal{B}_{n}$.

(ii) If $M \in \mathcal{B}_{n}$, then $M$ has a finite length as a left $A_{n}(K)$-module. In fact, every strictly increasing sequence of $A_{n}(K)$-modules contains at most $e(M)$ terms.

(iii) If $M$ is any filtered $A_{n}(K)$-module with an increasing filtration $\left(M_{r}\right)_{r \in \mathbf{Z}}$ and there exist positive integers $a$ and $b$ such that $\operatorname{dim}_{K} M_{r} \leqslant a r^{n}+$ $b(r+1)^{n-1}$ for all $r \in \mathbf{N}$, then $M \in \mathcal{B}_{n}$ and $e(M) \leqslant n ! a$.

\section{NUMERICAL POLYNOMIALS IN TWO VARIABLES}

Definition 3.1: A polynomial $f\left(t_{1}, t_{2}\right)$ in two variables $t_{1}$ and $t_{2}$ with rational coefficients is called numerical if $f\left(t_{1}, t_{2}\right) \in \mathbf{Z}$ for all sufficiently large $t_{1}, t_{2} \in \mathbf{Z}$, that is, there exists an element $\left(r_{0}, s_{0}\right) \in \mathbf{Z}^{2}$ such that $f(r, s) \in \mathbf{Z}$ for all integers $r \geqslant r_{0}, s \geqslant s_{0}$.

It is clear that every polynomial in two variables with integer coefficients is numerical. As an example of a numerical polynomial in two variables with noninteger coefficients one can consider a polynomial $\left(\begin{array}{l}t_{1} \\ m\end{array}\right)\left(\begin{array}{l}t_{2} \\ n\end{array}\right)$, where $m$ and $n$ are positive integers at least one of which is greater than 1 . (As usual, for any $k \in \mathbf{Z}, k \geqslant 1,\left(\begin{array}{l}t \\ k\end{array}\right)$ denotes the polynomial $\left(\begin{array}{l}t \\ k\end{array}\right)=t(t-1) \ldots(t-k+1) / k$ ! in one variable $t$; furthermore, we set $\left(\begin{array}{l}t \\ 0\end{array}\right)=1$, and $\left(\begin{array}{l}t \\ k\end{array}\right)=0$ if $k$ is a negative integer).

By the degree of a monomial $u=t_{1}^{i} t_{2}^{j}$ we mean its total degree $\operatorname{deg} u=i+j$, and the degrees of $u$ relative to $t_{1}$ and $t_{2}$ are defined as $\operatorname{deg}_{t_{1}} u=i$ and $\operatorname{deg}_{t_{2}} u=j$, 
respectively. If $f\left(t_{1}, t_{2}\right)=a_{1} u_{1}+\cdots+a_{k} u_{k}$ is a representation of a numerical polynomial $f\left(t_{1}, t_{2}\right)$ as a sum of monomials $u_{1}, \ldots, u_{k}$ with nonzero coefficients $a_{1}, \ldots, a_{k}$, then the degree of $f\left(t_{1}, t_{2}\right)$ and the degree of this polynomial relative to $t_{i}(i=1,2)$ are defined as usual: $\operatorname{deg} f=\max \left\{\operatorname{deg} u_{i} \mid 1 \leqslant i \leqslant k\right\}$ and $\operatorname{deg}_{t_{i}} f=\max \left\{\operatorname{deg}_{t_{i}} u_{i} \mid 1 \leqslant i \leqslant k\right\}$, respectively.

The following proposition proved in [9] gives a "canonical" representation of a numerical polynomial in two variables.

Proposition 3.1. Let $f\left(t_{1}, t_{2}\right)$ be a numerical polynomial in two variables $t_{1}, t_{2}$, and let $\operatorname{deg}_{t_{1}} f=p, \operatorname{deg}_{t_{2}} f=q$. Then the polynomial $f\left(t_{1}, t_{2}\right)$ can be represented in the form

$$
f\left(t_{1}, t_{2}\right)=\sum_{i=0}^{p} \sum_{j=0}^{q} a_{i j}\left(\begin{array}{c}
t_{1}+i \\
i
\end{array}\right)\left(\begin{array}{c}
t_{2}+j \\
j
\end{array}\right)
$$

with integer coefficients $a_{i j}(0 \leqslant i \leqslant p, 0 \leqslant j \leqslant q)$ that are uniquely defined by the polynomial $f\left(t_{1}, t_{2}\right)$.

In what follows (until the end of the section), we deal with subsets of the set $\mathbf{N}^{m+n}$ where $m$ and $n$ are positive integers. If $A \subseteq \mathbf{N}^{m+n}$, then $A(r, s)(r, s \in \mathbf{N})$ will denote the subset of $A$ that consists of all $(m+n)$-tuples $\left(a_{1}, \ldots, a_{m+n}\right)$ such that $a_{1}+\cdots+a_{m} \leqslant r$ and $a_{m+1}+\cdots+a_{m+n} \leqslant s$. Furthermore, $V_{A}$ will denote the set $\left\{v=\left(v_{1}, \ldots, v_{m+n}\right) \in \mathbf{N}^{m+n} \mid v\right.$ is not greater than or equal to any element of $A$ with respect to the product order on $\left.\mathbf{N}^{m+n}\right\}$. (Recall that the product order on the set $\mathbf{N}^{k}$ $(k \in \mathbf{N}, k \geqslant 1)$ is a partial order $\leqslant_{P}$ on $\mathbf{N}^{k}$ such that $\left(c_{1}, \ldots, c_{k}\right) \leqslant_{P}\left(c_{1}^{\prime}, \ldots, c_{k}^{\prime}\right)$ if and only if $c_{i} \leqslant c_{i}^{\prime}$ for all $i=1, \ldots, k$.) Clearly, an element $v=\left(v_{1}, \ldots, v_{m+n}\right) \in$ $\mathbf{N}^{m+n}$ belongs to $V_{A}$ if and only if for any element $\left(a_{1}, \ldots, a_{m+n}\right) \in A$ there exists $i \in \mathbf{N}, 1 \leqslant i \leqslant m+n$, such that $a_{i}>v_{i}$.

The following two statements proved in [10, Chapter II, Theorem 2.2.5 and Proposition 2.2.11] generalise the well-known Kolchin's result on numerical polynomials associated with subsets of $\mathbf{N}$ (see [8, Chapter 0 , Lemma 17]) and give the explicit formula for the numerical polynomials in two variables associated with a finite subset of $\mathbf{N}^{m+n}$ ( $m$ and $n$ are fixed positive integers).

Proposition 3.2. With the above notation, for any set $A \subseteq \mathbf{N}^{m+n}$, there exists a numerical polynomial $\omega_{A}\left(t_{1}, t_{2}\right)$ in two variables $t_{1}, t_{2}$ such that

(i) $\omega_{A}(r, s)=\operatorname{Card} V_{A}(r, s)$ for all sufficiently large $r, s \in \mathbf{N}$ (as usual, Card $V$ denotes the number of elements of a finite set $V$ );

(ii) $\operatorname{deg} \omega \leqslant m+n, \operatorname{deg}_{t_{1}} \omega \leqslant m$, and $\operatorname{deg}_{t_{2}} \omega \leqslant n$;

(iii) $\operatorname{deg} \omega=m+n$ if and only if the set $A$ is empty, in this case $\omega_{A}\left(t_{1}, t_{2}\right)=$ $\left(\begin{array}{c}t_{1}+m \\ m\end{array}\right)\left(\begin{array}{c}t_{2}+n \\ n\end{array}\right)$ 
(iv) $\omega_{A}\left(t_{1}, t_{2}\right)=0$ if and only if $(0,0) \in A$.

Definition 3.2: The polynomial $\omega_{A}\left(t_{1}, \ldots, t_{p}\right)$, whose existence is established by Proposition 3.2, is called the $(m, n)$-dimension polynomial of the set $A \subseteq \mathrm{N}^{m+n}$.

Proposition 3.3. Let $A=\left\{a_{1}, \ldots, a_{p}\right\}$ be a finite subset of $\mathrm{N}^{m+n}$ ( $m$ and $n$ are fixed positive integers) and let $a_{i}=\left(a_{i 1}, \ldots, a_{i, m+n}\right)$ for $i=1, \ldots, p$. Furthermore, for any $l \in \mathbf{N}, 0 \leqslant l \leqslant p$, let $\Gamma(l, p)$ denote the set of all $l$-element subsets of the set $\mathbf{N}_{p}=\{1, \ldots, p\}$, and for any $\sigma \in \Gamma(l, p)$ let $\bar{a}_{\sigma j}=\max \left\{a_{i j} \mid i \in \sigma\right\}$ $(1 \leqslant j \leqslant m+n), b_{\sigma}=\sum_{j=1}^{m} \bar{a}_{\sigma j}$, and $c_{\sigma}=\sum_{j=m+1}^{m+n} \bar{a}_{\sigma j}$. Then

$$
\omega_{A}\left(t_{1}, t_{2}\right)=\sum_{l=0}^{p}(-1)^{l} \sum_{\sigma \in \Gamma(l, p)}\left(\begin{array}{c}
t_{1}+m-b_{\sigma} \\
m
\end{array}\right)\left(\begin{array}{c}
t_{2}+n-c_{\sigma} \\
n
\end{array}\right) .
$$

Let $\mathbf{N}_{p}=\{1, \ldots, p\} \quad(p \in \mathbf{Z}, p \geqslant 1)$ be the set of the first $p$ positive integers and let $\mathbf{N}^{n} \times \mathbf{N}_{p}$ be the cartesian product of $n$ copies of $\mathbf{N}(n \in \mathbf{N})$ and $\mathbf{N}_{p}$ considered as an ordered set with respect to the product order $\leqslant P$ such that $\left(a_{1}, \ldots, a_{n}, b\right) \leqslant P\left(a_{1}^{\prime}, \ldots, a_{n}^{\prime}, b^{\prime}\right)$ if and only if $a_{i} \leqslant a_{i}^{\prime}$ for all $i=1, \ldots, n$ and $b \leqslant b^{\prime}$. As usual, if $\left(a_{1}, \ldots, a_{n}, b\right) \leqslant_{P}\left(a_{1}^{\prime}, \ldots, a_{n}^{\prime}, b^{\prime}\right)$ and $\left(a_{1}, \ldots, a_{m}, b\right) \neq\left(a_{1}^{\prime}, \ldots, a_{n}^{\prime}, b^{\prime}\right)$, we write $\left(a_{1}, \ldots, a_{n}, b\right)<_{P}\left(a_{1}^{\prime}, \ldots, a_{n}^{\prime}, b^{\prime}\right)$.

In what follows, we shall need the following result on the order $\leqslant P$ whose proof can be found in [8, Chapter 0 , Section 17]

LEMmA 3.4. Every infinite sequence of elements of $\mathbf{N}^{n} \times \mathbf{N}_{p}(n, p \in \mathbf{N}, p \geqslant 1)$ has an infinite subsequence, strictly increasing relative to the product order, in which every element has the same projection on $\mathbf{N}_{\boldsymbol{p}}$.

\section{REDUCTION IN FINITELY GENERATED FREE MODULES OVER WEYL ALGEBRAS. CHARACTERISTIC SETS}

The efficiency of the classical Gröbner basis methods for the computation of Hilbert polynomials of graded and filtered modules over polynomial rings is well-known. (One of the best presentations of the appropriate results and algorithms can be found in [1, Chapter 9] and [6, Section 15.10].) Similarly, the generalisation of the Gröbner basis technique to the rings of differential operators developed in [7] and [10, Chapter 4] allows us to find dimension polynomials of finitely generated differential modules (see [10, Chapter 4, Theorem 4.3.5]). In this section, proceeding in the spirit of the RittKolchin theory of characteristic sets (see [8, Chapter 1, Section $8-10$ and Chapter 2, Sections 12,13]), we generalise the classical Gröbner reduction to the case when the set of terms of a Weyl algebra $A_{n}(K)$ is considered together with two natural orderings. 
The results obtained allow us to prove the existence and give a method of computation of characteristic polynomials in two variables associated with a finite system of generators of an $A_{n}(K)$-module.

In what follows, we keep the notation and conventions of Section 2. In particular, $A_{n}(K)$ denotes a Weyl algebra in $n$ variables $x_{1}, \ldots, x_{n}$ over a field $K$ of zero characteristic, and the appropriate partial differentiations are denoted by $\partial_{1}, \ldots, \partial_{n}$, respectively. Furthermore, $\Theta$ will denote the commutative semigroup of all power products $x^{\alpha} \partial^{\beta}=x_{1}^{\alpha_{1}} \ldots x_{n}^{\alpha_{n}} \partial_{1}^{\beta_{1}} \ldots \partial_{n}^{\beta_{n}}$ with nonnegative integer exponents. If $\theta=x^{\alpha} \partial^{\beta} \in \Theta$, then the numbers $|\alpha|$ and $|\beta|$ will be called the orders of $\theta$ relative to the sets $\left\{x_{1}, \ldots, x_{n}\right\}$ and $\left\{\partial_{1}, \ldots, \partial_{n}\right\}$, respectively. These numbers will be denoted, respectively, by ord $\theta$ and ord $\partial \theta$.

Let $E$ be a finitely generated free $A_{n}(K)$-module with free generators $e_{1}, \ldots, e_{p}$. Obviously, $E$ can be considered as a vector $K$-space with the basis $\Theta e=\left\{\theta e_{i} \mid \theta \in\right.$ $\Theta, 1 \leqslant i \leqslant p\}$ whose elements will be called terms. For any term $\theta e_{j}$, we define the orders $\operatorname{ord}_{x}\left(\theta e_{j}\right)$ and $\operatorname{ord}_{\partial}\left(\theta e_{j}\right)$ of this term relative to the sets $\left\{x_{1}, \ldots, x_{n}\right\}$ and $\left\{\partial_{1}, \ldots, \partial_{n}\right\}$ as numbers $\operatorname{ord}_{x} \theta$ and $\operatorname{ord}_{\partial} \theta$, respectively. Furthermore, we say that a term $\theta e_{j}$ is a multiple of a term $\theta^{\prime} e_{k}$ if $\theta e_{j}=\theta^{\prime \prime}\left(\theta^{\prime} e_{k}\right)$ for some element $\theta^{\prime \prime} \in \Theta$. It will be written as $\theta^{\prime} e_{k} \mid \theta e_{j}$. (Of course, if $\theta^{\prime} e_{k} \mid \theta e_{j}$, then $k=j$ and $\theta^{\prime} \mid \theta$ in the semigroup $\Theta$.)

Below we consider two orders $<_{x}$ and $<_{\partial}$ on the set $\Theta e$ defined as follows: if $\theta e_{j}=x_{1}{ }^{i_{1}} \ldots x_{n}{ }^{i_{n}} \partial_{1}{ }^{j_{1}} \ldots \partial_{n}{ }^{j_{n}} e_{j}$ and $\theta^{\prime} e_{k}=x_{1}{ }^{k_{1}} \ldots x_{n}{ }^{k_{n}} \partial_{1}{ }^{l_{1}} \ldots \partial_{n}{ }^{l_{n}} e_{k}$, then $\theta e_{j}<_{x}$ $\theta^{\prime} e_{k}$ if and only if the vector $\left(\operatorname{ord}_{x} \theta, \operatorname{ord}_{\partial} \theta, j, i_{1}, \ldots, i_{n}, j_{1}, \ldots, j_{n}\right)$ is less than the vector $\left(\operatorname{ord}_{x} \theta^{\prime}, \operatorname{ord}_{\partial} \theta^{\prime}, k, k_{1}, \ldots, k_{n}, l_{1}, \ldots, l_{n}\right)$ with respect to the lexicographic order on $\mathbf{N}^{2 n+3}$; similarly, $\theta e_{j}<{ }_{\theta} \theta^{\prime} e_{k}$ if and only if $\left(\operatorname{ord}_{\partial} \theta, \operatorname{ord}_{x} \theta, j, j_{1}, \ldots, j_{n}, i_{1}, \ldots, i_{n}\right)$ is less than $\left(\operatorname{ord}_{y} \theta^{\prime}, \operatorname{ord}_{x} \theta^{\prime}, k, l_{1}, \ldots, l_{n}, k_{1}, \ldots, k_{n}\right)$ with respect to the lexicographic order on $\mathbf{N}^{2 n+3}$.

Since the set $\Theta e$ is a basis of the $K$-vector space $E$, every element $f \in E$ has a unique representation in the form

$$
f=a_{1} \theta_{1} e_{i_{1}}+\cdots+a_{q} \theta_{q} e_{i_{q}}
$$

where $\theta_{i} \in \Theta, a_{i} \in K, a_{i} \neq 0(1 \leqslant i \leqslant q), 1 \leqslant i_{1}, \ldots, i_{q} \leqslant p$, and $\theta_{r} e_{i_{r}} \neq \theta_{s} e_{i_{s}}$ if $r \neq s$.

Let $f$ be an element of the module $E$ written in the form (4.1) and let $\theta_{k} e_{i_{k}}$ and $\theta_{l} e_{i_{l}}$ be the greatest terms of the set $\left\{\theta_{1} e_{i_{1}}, \ldots, \theta_{q} e_{i_{q}}\right\}$ relative to the orders $<_{x}$ and $<_{\partial}$, respectively. (It is possible that $k=l$, that is, $\theta_{k} e_{i_{k}}=\theta_{l} e_{i_{l}}$ ). Then the terms $\theta_{k} e_{i_{k}}$ and $\theta_{l} e_{i_{l}}$ are called the $x$-leader and $\partial$-leader of the element $f$; they are denoted by $u_{f}$ and $v_{f}$, respectively. The coefficients of the $x$ - and $\partial$-leaders of an element $f \in E$ will be denoted by $l c_{x}(f)$ and $l c_{\partial}(f)$, respectively. 
Let $f$ and $g$ be two elements of the free $R$-module $E$ considered above. The element $f$ is said to be reduced with respect to $g$ if $f$ does not contain any multiple $\theta u_{g}(\theta \in \Theta)$ of the $x$-leader $u_{g}$ such that $\operatorname{ord}_{\partial}\left(\theta v_{g}\right) \leqslant \operatorname{ord}_{\partial} v_{f}$. (We say that an element $f \in E$ contains a term $\theta e_{j}$ if this term appears in representation (4.1) with a nonzero coefficient.) An element $h \in E$ is said to be reduced with respect to a set $\Sigma \subseteq E$, if $h$ is reduced with respect to every element of the set $\Sigma$.

Definition 4.1: A subset $\Sigma$ of the free $A_{n}(K)$-module $E$ is called autoreduced if every element of $\Sigma$ is reduced with respect to any other element of this set.

The following statement is the direct consequence of Lemma 3.4.

LEMMA 4.1. Let $E$ be the free $A_{n}(K)$-module considered above and let $S$ be an infinite sequence of terms from the set $\Theta e$. Then there exists an index $j(1 \leqslant j \leqslant p)$ and an infinite subsequence $\theta_{1} e_{j}, \theta_{2} e_{j}, \ldots, \theta_{k} e_{j}, \ldots$ of the sequence $S$ such that $\theta_{k} \mid \theta_{k+1}$ for all $k=1,2, \ldots$.

PROPOSITION 4.2. Every autoreduced subset of the free $A_{n}(K)$-module $E$ is finite.

Proof: Let $\Sigma$ be an autoreduced subset of $E$. First of all, note that if $f, g \in \Sigma$ and $f \neq g$, then $u_{f} \neq u_{g}$. Indeed, since the elements $f$ and $g$ are reduced with respect to each other, the equality $u_{f}=u_{g}$ would imply that $\operatorname{ord}_{\partial} v_{g}<\operatorname{ord}_{\partial} v_{f}$ and $\operatorname{ord}_{\partial} v_{f}<\operatorname{ord}_{\partial} v_{g}$ at the same time.

Suppose that our autoreduced set $\Sigma$ is infinite. Then the set $U=\left\{u_{f} \mid f \in \Sigma\right\}$ is infinite and it does not contain two equal elements. By Lemma 4.1, there exists an infinite sequence $u_{f_{1}}, u_{f_{2}}, \ldots$ of elements of $U$ such that $u_{f_{i}} \mid u_{f_{i+1}}$ for all $i=1,2, \ldots$, that is, $u_{f_{i+1}}=\theta_{i} u_{f_{i}}$ for some $\theta_{i} \in \Theta(i=1,2, \ldots)$. Let $k_{i}=\operatorname{ord}_{\partial} u_{f_{i}}$ and $l_{i}=$ $\operatorname{ord}_{\partial} v_{f_{i}}(i=1,2, \ldots)$. It is clear that if $l_{i+1}-l_{i} \geqslant k_{i+1}-k_{i}$ for some index $i$, then $f_{i+1}$ is not reduced with respect to $f_{i}$, so we should have $l_{i+1}-l_{i}<k_{i+1}-k_{i}$ for all $i=1,2 \ldots$ Therefore, $l_{i+1}-k_{i+1}<l_{i}-k_{i}$ for all $i=1,2, \ldots$ This contradicts the fact that $l_{i} \geqslant k_{i}$ for all $i=1,2, \ldots$.

PRoposition 4.3. Let $\Sigma=\left\{g_{1}, \ldots, g_{r}\right\}$ be an autoreduced subset of the free $R$-module $E$ and let $f \in E$. Then there exists an element $g \in E$ such that $f-g=\sum_{i=1}^{r} \lambda_{i} g_{i}$ for some $\lambda_{1}, \ldots, \lambda_{r} \in A_{n}(K)$ and $g$ is reduced with respect to the set $\Sigma$.

Proof: If $f$ is reduced with respect to $\Sigma$, the statement is obvious (one can set $g=f)$. Suppose that $f$ is not reduced with respect to $\Sigma$. Let $u_{i}$ and $v_{i}$ be the leaders of the element $g_{i}$ relative to the orders $<_{x}$ and $<_{\theta}$, respectively, and let $a_{i}$ be the coefficient of the term $u_{i}$ in $g_{i}(i=1, \ldots, r)$. In what follows, a term $w_{h}$, that appears in an element $h \in E$, will be called a $\Sigma$-leader of $h$ if $w_{h}$ is the greatest (with respect 
to the order $\left.<_{x}\right)$ term among all terms $\theta u_{j}(\theta \in \Theta, 1 \leqslant j \leqslant r)$ that appear in $h$ and satisfy the condition ord $\left(\theta v_{j}\right) \leqslant \operatorname{ord}_{\partial} v_{h}$. (As above, $u_{h}$ and $v_{h}$ denote the leaders of the element $h$ relative to the orders $<_{x}$ and $<_{\partial}$, respectively).

Let $w_{f}$ be the $\Sigma$-leader of the element $f$ and let $c_{f}$ be the coefficient of $w_{f}$ in $f$. Then $w_{f}=\theta u_{j}$ for some $\theta \in \Theta$, and for some $j(1 \leqslant j \leqslant r)$ such that $\operatorname{ord}_{\partial}\left(\theta v_{j}\right) \leqslant \operatorname{ord}_{\partial} v_{f}$. Without loss of generality we may assume that $j$ corresponds to the maximum (with respect to the order $<_{x}$ ) $x$-leader $u_{j}$ in the set of all $x$-leaders of elements of $\Sigma$. Let us consider the element $f^{\prime}=f-\left(c_{f} / a_{j}\right) \theta g_{j}$. Obviously, $f^{\prime}$ does not contain $w_{f}$ and ord $\partial\left(v_{f^{\prime}}\right) \leqslant \operatorname{ord}_{\partial} v_{f}$. Furthermore, $f^{\prime}$ cannot contain any term of the form $\theta^{\prime} u_{i}\left(\theta^{\prime} \in \Theta, 1 \leqslant i \leqslant r\right)$ that is greater than $w_{f}$ (with respect to $<_{x}$ ) and satisfies the condition $\operatorname{ord}_{\partial}\left(\theta^{\prime} v_{i}\right) \leqslant \operatorname{ord}_{\partial} v_{f^{\prime}}$. Indeed, if the last inequality holds, then $\operatorname{ord}_{\partial}\left(\theta^{\prime} v_{i}\right) \leqslant \operatorname{ord}_{\partial} v_{f}$, so that the term $\theta^{\prime} u_{i}$ cannot appear in $f$. This term cannot appear in $\theta g_{j}$ either, since $u_{\theta g_{j}}=\theta u_{j}=w_{f}<_{x} \theta^{\prime} u_{i}$. Thus, $\theta^{\prime} u_{i}$ cannot appear in $f^{\prime}=f-\left(c_{f} / a_{j}\right) \theta g_{j}$, whence the $\Sigma$-leader of $f^{\prime}$ is strictly less (with respect to the order $<_{x}$ ) than the $\Sigma$-leader of $f$. Applying the same procedure to $f^{\prime}$ and continuing in the same way, we obtain an element $g \in E$ such that $f-g$ is a linear combination of elements $g_{1}, \ldots, g_{r}$ with coefficients from $A_{n}(K)$ and $g$ is reduced with respect to $\Sigma$.

DEFINITION 4.2: Let $f$ and $g$ be two elements of the free $A_{n}(K)$-module $E$. We say that the element $f$ has lower rank than $g$ and write $r k(f)<r k(g)$ if either $u_{f}<_{x} u_{g}$ or $u_{f}=u_{g}$ and $v_{f}<_{\partial} v_{g}$. If $u_{f}=u_{g}$ and $v_{f}=v_{g}$, we say that $f$ and $g$ have the same rank and write $r k(f)=r k(g)$.

In what follows, while considering autoreduced subsets of $E$, we always assume that their elements are arranged in order of increasing rank. (Therefore, if we consider an autoreduced set $\Sigma=\left\{h_{1}, \ldots, h_{r}\right\} \subseteq E$, then $\left.r k\left(h_{1}\right)<\cdots<r k\left(h_{r}\right)\right)$.

Definition 4.3: Let $\Sigma=\left\{h_{1}, \ldots, h_{r}\right\}$ and $\Sigma^{\prime}=\left\{h_{1}^{\prime}, \ldots, h_{s}^{\prime}\right\}$ be two autoreduced subsets of the free $A_{n}(K)$-module $E$. An autoreduced set $\Sigma$ is said to have lower rank than $\Sigma^{\prime}$ if one of the following two cases holds:

(1) There exists $k \in \mathbf{N}$ such that $1 \leqslant k \leqslant \min \{r, s\}, r k\left(h_{i}\right)=r k\left(h_{i}^{\prime}\right)$ for $i=1, \ldots, k-1$ and $r k\left(h_{k}\right)<r k\left(h_{k}^{\prime}\right)$.

(2) $\quad r>s$ and $r k\left(h_{i}\right)=r k\left(h_{i}^{\prime}\right)$ for $i=1, \ldots, s$.

If $r=s$ and $r k\left(h_{i}\right)=r k\left(h_{i}^{\prime}\right)$ for $i=1, \ldots, r$, then $\Sigma$ is said to have the same rank as $\Sigma^{\prime}$.

PROPOSITION 4.4. In every nonempty set of autoreduced subsets of the free $A_{n}(K)$-module $E$ there exists an autoreduced subset of lowest rank.

Proof: Let $\Phi$ be any nonempty set of autoreduced subsets of $E$. Define by induction an infinite descending chain of subsets of $\Phi$ as follows: $\Phi_{0}=\Phi, \Phi_{1}=\{\Sigma \in$ 
$\Phi_{0} \mid \Sigma$ contains at least one element and the first element of $\Sigma$ is of lowest possible rank $\}, \ldots, \Phi_{k}=\left\{\Sigma \in \Phi_{k-1} \mid \Sigma\right.$ contains at least $k$ elements and the $k$ th element of $\Sigma$ is of lowest possible rank\}, ... Obviously, if $\Phi_{k} \neq \emptyset$, then $k$ th elements of autoreduced sets from $\Phi_{k}$ have the same $x$-leader $u_{k}$ and the same $y$-leader $v_{k}$. If $\Phi_{k}$ were nonempty for all $k=1,2, \ldots$, the set $\left\{f_{k} \mid f_{k}\right.$ is the $k$ th element of some autoreduced set from $\Phi_{k}$ \} would be an infinite autoreduced set, which would contradict Proposition 4.2. Therefore, there is a smallest $k$ such that $\Phi_{k}=\emptyset$. (Since, $\Phi_{0}=\Phi$ is nonempty, $k>0$ ). It is clear that every element of $\Phi_{k-1}$ is an autoreduced subset in $\Phi$ of lowest rank.

Definition 4.4: Let $N$ be a $A_{n}(K)$-submodule of the free $A_{n}(K)$-module $E$. An autoreduced subset of $N$ of lowest rank is called a characteristic set of the module $N$.

Proposition 4.5. Let $N$ be an $A_{n}(K)$-submodule of the free $A_{n}(K)$ module $E$ and let $\Sigma=\left\{g_{1}, \ldots, g_{r}\right\}$ be a characteristic set of $N$. Then an element $f \in N$ is reduced with respect to $\Sigma$ if and only if $f=0$.

Proof: Suppose that $f$ is a nonzero element of $N$ reduced with respect to $\Sigma$. If $r k(f)<r k\left(g_{1}\right)$, then the autoreduced set $\{f\}$ has lower rank than $\Sigma$. If $r k\left(g_{1}\right)<r k(f)$ ( $f$ and $g_{1}$ cannot have the same rank, since $f$ is reduced with respect to $\Sigma$ ), then $f$ and the elements $g \in \Sigma$ that have lower rank than $f$ form an autoreduced set that has lower rank than $\Sigma$. In both cases we arrive at a contradiction with the fact that $\Sigma$ is a characteristic set of $N$.

Proposition 4.6. Let $N$ be a cyclic $A_{n}(K)$-submodule of the free $R$-module $E$ generated by an element $g \in E$. Then $\{g\}$ is a characteristic set of the module $N=A_{n}(K) g$.

Proof: Let $h \in N$, so that $h$ can be written as $h=\sum_{i=1}^{k} c_{i} \theta_{i} g$ where $\theta_{i} \in \Theta$, $c_{i} \in K, c_{i} \neq 0(1 \leqslant i \leqslant k)$, and $\theta_{i} \neq \theta_{j}$ if $i \neq j(1 \leqslant i, j \leqslant k)$. Obviously, if $\theta_{p}$ and $\theta_{\boldsymbol{q}}$ are maximal elements of the set $\left\{\theta_{1}, \ldots, \theta_{k}\right\}$ relative to the orders $<_{x}$ and $<_{\partial}$, respectively, then $u_{h}=\theta_{p} u_{g}$ and $v_{h}=\theta_{q} v_{g}$. We see that $h$ contains the multiple $\theta_{p} u_{g}$ of the $x$-leader $u_{g}$ such that $\operatorname{ord}_{\partial}\left(\theta_{p} v_{g}\right) \leqslant \operatorname{ord}_{\theta}\left(\theta_{q} v_{g}\right)=\operatorname{ord}_{\partial} v_{h}$ whence $h$ is not reduced with respect to $g$. Furthermore, since $u_{g} \leqslant_{x} \theta_{p} u_{g}=u_{h}$ and $v_{g} \leqslant_{a} \theta_{q} v_{g}=v_{h}$, $r k(g) \leqslant r k(h)$, and $r k(g)=r k(h)$ if and only if $\theta_{p}=\theta_{q}=1$, that is $h=c g$ for some $c \in K$. Thus, $N$ does not contain elements reduced with respect to $g$, and $g$ is the element of the lowest rank in $N$. It follows that if $\Sigma=\left\{h_{1}, \ldots, h_{l}\right\}$ is a characteristic set of $N$, then $r k(g)=r k\left(h_{1}\right)$ and $l=1$, whence $\{g\}$ is also a characteristic set of $N$.

Proposition 4.7. Let $N$ be an $A_{n}(K)$-submodule of the free $A_{n}(K)$ - 
module $E$ and let $\Sigma=\left\{g_{1}, \ldots, g_{r}\right\}$ be a characteristic set of $N$. Then the elements $g_{1}, \ldots, g_{r}$ generate the $A_{n}(K)$-module $N$.

ProOF: Let $f$ be any element of $N$. By Proposition 4.3, there exist elements $\lambda_{1}, \ldots, \lambda_{r} \in A_{n}(K)$ and an element $f^{\prime} \in E$ such that $f^{\prime}$ is reduced with respect to $\Sigma$ and $f-f^{\prime}=\sum_{i=1}^{r} \lambda_{i} g_{i}$. Therefore, $f^{\prime} \in N$, and Proposition 4.5 shows that $f^{\prime}=0$, whence $f=\sum_{i=1}^{r} \lambda_{i} g_{i}$.

Proposition 4.8. Let $N$ be an $A_{n}(K)$-submodule of the free $A_{n}(K)$ module $E$ and let $\Sigma_{1}=\left\{g_{1}, \ldots, g_{r}\right\}$ and $\Sigma_{2}=\left\{h_{1}, \ldots, h_{s}\right\}$ be two characteristic sets of $N$ such that $l c_{x}\left(g_{i}\right)=1$ and $l c_{x}\left(h_{j}\right)=1$ for $i=1, \ldots, r ; j=1, \ldots, s$. Then $r=s$ and $g_{i}=h_{i}$ for $i=1, \ldots, r$.

PROOF: Since $\Sigma_{1}$ and $\Sigma_{2}$ are two autoreduced sets of the same (lowest possible) rank, $r=s, u_{g_{i}}=u_{h_{i}}$, and $v_{g_{i}}=v_{h_{i}}$ for $i=1, \ldots, r$. Suppose that there exists $i$, $1 \leqslant i \leqslant r$, such that $g_{i} \neq h_{i}$. Setting $f_{i}=g_{i}-h_{i}$ we obtain that $u_{f_{i}}<_{x} u_{g_{i}}$ (since $\left.l c_{x}\left(g_{i}\right)=l c_{x}\left(h_{i}\right)=1\right), v_{f_{i}} \leqslant y v_{g_{i}}$, and $f_{i}$ is reduced with respect to any element $g_{j}$ $(1 \leqslant j \leqslant r)$. Indeed, suppose that $f_{i}$ contains a multiple $\theta u_{g_{j}}$ of some $x$-leader $u_{g_{j}}$ such that $\operatorname{ord}_{\partial}\left(\theta v_{g_{j}}\right) \leqslant \operatorname{ord}_{\partial} v_{f_{i}}$ (obviously, $f_{i}$ is reduced with respect to $g_{i}$, so we can assume that $j \neq i$ ). Then at least one of the elements $g_{i}, h_{i}$ must contain $\theta u_{g_{j}}$ and $\operatorname{ord}_{\partial}\left(\theta v_{g_{j}}\right)=\operatorname{ord}_{\partial}\left(\theta v_{h_{j}}\right) \leqslant \operatorname{ord}_{y} v_{f_{i}} \leqslant \operatorname{ord}_{y} v_{g_{i}}=\operatorname{ord}_{y} v_{h_{i}}$, which contradicts the fact that the sets $\Sigma_{1}$ and $\Sigma_{2}$ are autoreduced. Now, Proposition 4.5 shows that $f_{i}=0$ whence $g_{i}=h_{i}$.

\section{Bifiltered modules over Weyl algebras, their Characteristic POLYNOMIALS AND INVARIANTS}

In what follows, the ring $A_{n}(K)$ will be considered as a bifiltered ring with respect to the natural bifiltration defined as a bisequence $\left(D_{r s}\right)_{r, s \in Z}$ such that $D_{r s}=0$, if at least one of the numbers $r, s$ is negative, and if $r \geqslant 0, s \geqslant 0$, then $D_{r s}$ is a vector $K$-space generated by the set $\Theta(r, s)=\left\{\theta \in \Theta \mid \operatorname{ord}_{x} \theta \leqslant r, \operatorname{ord}_{\partial} \theta \leqslant s\right\}$. Obviously, $\bigcup\left\{D_{r s} \mid r, s \in \mathbf{Z}\right\}=A_{n}(K), D_{r s} \subseteq D_{r+1, s}, D_{r s} \subseteq D_{r, s+1}$ for any $r, s \in \mathbf{Z}$, and $D_{k l} D_{r s}=D_{r+k, s+l}$ for any $r, s, k, l \in \mathrm{N}$. Furthermore, as follows from the third statement of Proposition 3.2, Card $\Theta(r, s)=\left(\begin{array}{c}r+n \\ m\end{array}\right)\left(\begin{array}{c}s+n \\ n\end{array}\right)$ for any $r, s \in \mathbf{N}$.

DEFINITION 5.1: Let $M$ be a module over a Weyl algebra $A_{n}(K)$ equipped with the bifiltration $\left(D_{r s}\right)_{r, s \in \mathbf{Z}}$. A bisequence $\left(M_{r s}\right)_{r, s \in Z}$ of vector $K$-subspaces of the module $M$ is called a bifiltration of $M$ if the following three conditions hold:

(i) If $r \in \mathbf{Z}$ is fixed, then $M_{r s} \subseteq M_{r, s+1}$ for all $s \in \mathbf{Z}$ and $M_{r s}=\mathbf{0}$ for all sufficiently small $s \in \mathbf{Z}$. Similarly, if $s \in \mathbf{Z}$ is fixed, then $M_{r s} \subseteq M_{r+1, s}$ 
for all $r \in \mathbf{Z}$ and $M_{r s}=0$ for all sufficiently small $r \in \mathbf{Z}$.

(ii) $\bigcup\left\{M_{r s} \mid r, s \in \mathbf{Z}\right\}=M$.

(iii) $D_{k l} M_{r s} \subseteq M_{r+k, s+l}$ for any $r, s \in \mathbf{Z}, k, l \in \mathbf{N}$.

EXAMPLE 5.1. Let $M$ be a finitely generated $A_{n}(K)$-module with generators $f_{1}, \ldots, f_{p}$. Then the vector $K$-spaces $M_{r s}=\sum_{i=1}^{p} D_{r s} f_{i}(r, s \in \mathbf{Z})$ form a bifiltration of the module $M$. This bifiltration is called the natural bifiltration of $M$ associated with the system of generators $f_{1}, \ldots, f_{p}$.

It is easy to see that every component $M_{r s}$ of the natural bifiltration is a finitely generated vector $K$-space and $D_{k l} M_{r s}=M_{r+k, s+l}$ for any $r, s, k, l \in \mathrm{N}$.

THEOREM 5.1. Let $M$ be a finitely generated $A_{n}(K)$-module with a system of generators $\left\{f_{1}, \ldots, f_{p}\right\}, E$ a free $A_{n}(K)$-module with a basis $e_{1}, \ldots, e_{p}$, and $\pi$ : $E \longrightarrow M$ the natural $A_{n}(K)$-epimorphism of $E$ onto $M\left(\pi\left(e_{i}\right)=f_{i}\right.$ for $\left.i=1, \ldots, p\right)$. Furthermore, let $N=\operatorname{Ker} \pi$ and let $\Sigma=\left\{g_{1}, \ldots, g_{d}\right\}$ be a characteristic set of $N$. Finally, for any $r, s \in \mathbf{N}$, let $M_{r s}=\sum_{i=1}^{p} D_{r s} f_{i}$, and let $U_{r s}$ denote the set $\{w \in \Theta e \mid$ $\operatorname{ord}_{x} w \leqslant r, \operatorname{ord}_{\partial} w \leqslant s$, and either $w$ is not a multiple of any $u_{g_{i}}(1 \leqslant i \leqslant d)$ or $\operatorname{ord}_{\partial}\left(\theta v_{g_{j}}\right)>s$ for any $\theta \in \Theta, g_{j} \in \Sigma$ such that $\left.w=\theta u_{g_{j}}\right\}$.

Then $\pi\left(U_{r s}\right)$ is a basis of the vector $K$-space $M_{r s}$.

Proof: Let us prove, first, that every element $\theta f_{i}(1 \leqslant i \leqslant p, \theta \in \Theta(r, s))$, that does not belong to $\pi\left(U_{r s}\right)$, can be written as a finite linear combination of elements of $\pi\left(U_{r s}\right)$ with coefficients from $K$ (so that the set $\pi\left(U_{r s}\right)$ generates the vector $K$-space $\left.M_{r s}\right)$. Since $\theta f_{i} \notin \pi\left(U_{r s}\right), \theta e_{i} \notin U_{r s}$ whence $\theta e_{i}=\theta^{\prime} u_{g_{j}}$ for some $\theta^{\prime} \in \Theta, 1 \leqslant j \leqslant d$, such that $\operatorname{ord}_{\partial}\left(\theta^{\prime} v_{g_{j}}\right) \leqslant s$. Let us consider the element $g_{j}=a_{j} u_{g_{j}}+\ldots\left(a_{j} \in K\right.$, $a_{j} \neq 0$ ), where dots are placed instead of the other terms that appear in $g_{j}$ (obviously, those terms are less than $u_{g_{j}}$ with respect to the order $\left.<_{x}\right)$. Since $g_{j} \in N=\operatorname{Ker} \pi$, $\pi\left(g_{j}\right)=a_{j} \pi\left(u_{g_{j}}\right)+\cdots=0$, whence $\pi\left(\theta^{\prime} g_{j}\right)=a_{j} \pi\left(\theta^{\prime} u_{g_{j}}\right)+\cdots=a_{j} \pi\left(\theta e_{i}\right)+\cdots=$ $a_{j} \theta f_{i}+\cdots=0$, so that $\theta f_{i}$ is a finite linear combination with coefficients from $K$ of some elements $\tilde{\theta} f_{k}(1 \leqslant k \leqslant p)$ such that $\tilde{\theta} \in \Theta(r, s)$ and $\tilde{\theta} e_{k}<_{x} \theta^{\prime} u_{g_{j}}$. (Note $\operatorname{ord}_{x} \tilde{\theta} \leqslant r$, since $\tilde{\theta} e_{k}<_{x} \theta e_{i}$ and $\theta \in \Theta(r, s) ; \operatorname{ord}_{\partial} \tilde{\theta} \leqslant s$, since $\tilde{\theta} e_{k} \leqslant \partial v_{\theta^{\prime} g_{j}}=\theta^{\prime} v_{g_{j}}$ and $\left.\operatorname{ord}_{\partial}\left(\theta^{\prime} v_{g_{j}}\right) \leqslant s\right)$. Thus, we can apply induction on $\theta e_{j}(\theta \in \Theta, 1 \leqslant j \leqslant p)$ with respect to the order $<_{x}$ and obtain that every element $\theta f_{i}(\theta \in \Theta(r, s), 1 \leqslant j \leqslant p)$ can be written as a finite linear combination of elements of $\pi\left(U_{r s}\right)$ with coefficients from the field $K$.

Now, let us prove that the set $\pi\left(U_{r s}\right)$ is linearly independent over $K$. Let $\sum_{i=1}^{q} a_{i} \pi\left(u_{i}\right)=0$ for some $u_{1}, \ldots, u_{q} \in U_{r s}, a_{1}, \ldots, a_{q} \in K$. Then $h=\sum_{i=1}^{q} a_{i} u_{i}$ is an ele- 
ment of $N$ reduced with respect to $\Sigma$. Indeed, if an element $u=\theta e_{j}$ appears in $h$ (so that $u=u_{i}$ for some $\left.i=1, \ldots, q\right)$, then either $u$ is not a multiple of any $u_{g_{j}}(1 \leqslant j \leqslant d)$ or $u=\theta u_{g_{k}}$ for some $\theta \in \Theta, 1 \leqslant k \leqslant d$, such that $\operatorname{ord}_{\partial}\left(\theta v_{g_{k}}\right)>s \geqslant \operatorname{ord}_{\partial} v_{h}$ (since $v_{h}$ is one of the elements $u_{1}, \ldots, u_{q}$ that lie in $\left.U_{r s}\right)$. Applying Proposition 4.5, we obtain that $h=0$, whence $a_{1}=\cdots=a_{q}=0$. This completes the proof of the theorem.

The following theorem is the main result of this section.

Theorem 5.2. Let $M$ be a finitely generated $A_{n}(K)$-module with a system of generators $\left\{f_{1}, \ldots, f_{p}\right\}$ and let $\left(M_{r s}\right)_{r, s \in \mathbf{N}}$ be the corresponding natural bifiltration of $M\left(M_{r s}=\sum_{i=1}^{p} D_{r s} f_{i}\right.$ for $\left.r, s \in \mathbf{N}\right)$. Then there exists a numerical polynomial $\phi_{M}\left(t_{1}, t_{2}\right)$ in two variables $t_{1}, t_{2}$ such that $\operatorname{deg}_{t_{1}} \phi_{M}\left(t_{1}, t_{2}\right) \leqslant n, \operatorname{deg}_{t_{2}} \phi_{M}\left(t_{1}, t_{2}\right) \leqslant n$, and $\phi_{M}(r, s)=\operatorname{dim}_{K} M_{r s}$ for all sufficiently large $(r, s) \in \mathrm{N}^{2}$.

Proof: Let $E$ be a free $A_{n}(K)$-module with a basis $e_{1}, \ldots, e_{p}$, let $N$ be the kernel of the natural epimorphism $\pi: E \longrightarrow M$, and let the set $U_{r s}(r, s \in \mathbf{N})$ be the same as in the conditions of Theorem 5.1. (As before, $\Sigma=\left\{g_{1}, \ldots, g_{d}\right\}$ is a characteristic set of $N$.) By Theorem 5.1, for any $r, s \in \mathbf{N}, \pi\left(U_{r s}\right)$ is a basis of the vector $K$-space $M_{r s}$. Therefore, $\operatorname{dim}_{K} M_{r s}=\operatorname{Card} \pi\left(U_{r s}\right)=\operatorname{Card} U_{r s}$. (It was shown in the second part of the proof of Theorem 5.1 that the restriction of the mapping $\pi$ on $U_{r s}$ is bijective.)

Let $U_{r s}^{\prime}=\left\{w \in U_{r s} \mid w\right.$ is not a multiple of any element $\left.u_{g_{i}}(1 \leqslant i \leqslant d)\right\}$ and $U_{r s}^{\prime \prime}=\left\{w \in U_{r s} \mid w=\theta u_{g_{j}}\right.$ for some $g_{j}(1 \leqslant j \leqslant d)$ and $\theta \in \Theta$ such that $\operatorname{ord}_{\partial}\left(\theta v_{g_{j}}\right)>$ $s\}$. Then $U_{r s}=U_{r s}^{\prime} \cup U_{r s}^{\prime \prime}$ and $U_{r s}^{\prime} \cap U_{r s}^{\prime \prime}=\emptyset$, whence Card $U_{r s}=\operatorname{Card} U_{r s}^{\prime}+\operatorname{Card} U_{r s}^{\prime \prime}$.

By Proposition 3.2, there exists a numerical polynomial $\omega\left(t_{1}, t_{2}\right)$ in two variables $t_{1}$ and $t_{2}$ such that $\omega(r, s)=\operatorname{Card} U_{r s}^{\prime}$ for all sufficiently large $(r, s) \in$ $\mathbf{N}^{2}$. In order to express Card $U_{r s}^{\prime \prime}$ in terms of $r$ and $s$, let us set $a_{i}=\operatorname{ord}_{x} u_{g_{i}}$, $b_{i}=\operatorname{ord}_{\partial} u_{g_{i}}, c_{i}=\operatorname{ord}_{\partial} v_{g_{i}}, a_{i j}=\operatorname{ord}_{x} l c m\left(u_{g_{i}}, u_{g_{j}}\right), b_{i j}=\operatorname{ord} \partial l c m\left(u_{g_{i}}, u_{g_{j}}\right)$, $a_{i j k}=\operatorname{ord}_{x} l c m\left(u_{g_{i}}, u_{g_{j}}, u_{g_{k}}\right), b_{i j k}=\operatorname{ord}{ }_{\partial} l c m\left(u_{g_{i}}, u_{g_{j}}, u_{g_{k}}\right), \ldots(1 \leqslant i, j, k, \ldots \leqslant d)$. Then $U_{r s}^{\prime \prime}=\bigcup_{i=1}^{d}\left\{\left[\Theta\left(r-a_{i}, s-b_{i}\right) \backslash \Theta\left(r-a_{i}, s-c_{i}\right)\right] u_{g_{i}}\right\}$. By the combinatorial principle of inclusion and exclusion (see [5, Chapter 5, Theorem 5.1.1]),

$$
\begin{array}{r}
\operatorname{Card} U_{r s}^{\prime \prime}=\sum_{i=1}^{d} \operatorname{Card}\left\{\left[\Theta\left(r-a_{i}, s-b_{i}\right) \backslash \Theta\left(r-a_{i}, s-c_{i}\right)\right] u_{g_{i}}\right\} \\
-\sum_{1 \leqslant i<j \leqslant d} \operatorname{Card}\left\{\left[\Theta\left(r-a_{i}, s-b_{i}\right) \backslash \Theta\left(r-a_{i}, s-c_{i}\right)\right] u_{g_{i}}\right. \\
\left.\cap\left[\Theta\left(r-a_{j}, s-b_{j}\right) \backslash \Theta\left(r-a_{j}, s-c_{j}\right)\right] u_{g_{j}}\right\}
\end{array}
$$




$$
\begin{aligned}
+\sum_{1 \leqslant i<j<k \leqslant d} \operatorname{Card} & \left\{\left[\Theta\left(r-a_{i}, s-b_{i}\right) \backslash \Theta\left(r-a_{i}, s-c_{i}\right)\right] u_{g_{i}}\right. \\
& \cap\left[\Theta\left(r-a_{j}, s-b_{j}\right) \backslash \Theta\left(r-a_{j}, s-c_{j}\right)\right] u_{g_{j}} \\
& \left.\cap\left[\Theta\left(r-a_{k}, s-b_{k}\right) \backslash \Theta\left(r-a_{k}, s-c_{k}\right)\right] u_{g_{k}}\right\}-\ldots
\end{aligned}
$$

Furthermore, for any two different elements $g_{i}, g_{j} \in \Sigma$, we have

$$
\begin{gathered}
\operatorname{Card}\left\{\left[\Theta\left(r-a_{i}, s-b_{i}\right) \backslash \Theta\left(r-a_{i}, s-c_{i}\right)\right] u_{g_{i}}\right. \\
\left.\cap\left[\Theta\left(r-a_{j}, s-b_{j}\right) \backslash \Theta\left(r-a_{j}, s-c_{j}\right)\right] u_{g_{j}}\right\} \\
=\operatorname{Card}\left\{\theta l c m\left(u_{g_{i}}, u_{g_{j}}\right) \mid \theta \in \Theta, \operatorname{ord}_{x} \theta \leqslant r-a_{i j}, \operatorname{ord}_{\partial} \theta \leqslant s-b_{i j},\right. \\
\quad \operatorname{ord}_{\partial}\left(\theta\left(l c m\left(u_{g_{i}}, u_{g_{j}}\right) /\left(u_{g_{i}}\right)\right) v_{g_{i}}\right)=\operatorname{ord}_{\partial} \theta+b_{i j}-b_{i}+c_{i}>s \\
\left.\quad \operatorname{and~} \operatorname{ord}_{\partial} \theta+b_{i j}-b_{j}+c_{j}>s\right\} \\
=\operatorname{Card} \cdot\left\{\theta \mid \theta \in \Theta, \operatorname{ord}_{x} \theta \leqslant r-a_{i j}, \operatorname{ord}_{\partial} \theta \leqslant s-b_{i j}\right. \text { and } \\
\quad \operatorname{ord}_{\partial} \theta>s-\min _{2}\left\{c_{i}+b_{i j}-a_{i}, c_{j}+b_{i j}-a_{j}\right\} \\
=\left(\begin{array}{c}
r+n-a_{i j} \\
n
\end{array}\right)\left[\left(\begin{array}{c}
s+n-b_{i j} \\
n
\end{array}\right)-\left(s+n-\min \left\{c_{i}+b_{i j}-b_{i}, c_{j}+b_{i j}-b_{j}\right\}\right)\right] . \\
n
\end{gathered}
$$

Similarly, for any three different elements $g_{i}, g_{j}, g_{k} \in \Sigma$ we obtain that

$$
\begin{aligned}
\operatorname{Card}\left\{\left[\Theta\left(r-a_{i}, s-b_{i}\right) \backslash \Theta\left(r-a_{i}, s-c_{i}\right)\right] u_{g_{i}}\right. \\
\cap\left[\Theta\left(r-a_{j}, s-b_{j}\right) \backslash \Theta\left(r-a_{j}, s-c_{j}\right)\right] u_{g_{j}} \\
\left.\cap\left[\Theta\left(r-a_{k}, s-b_{k}\right) \backslash \Theta\left(r-a_{k}, s-c_{k}\right)\right] u_{g_{k}}\right\} \\
=\left(\begin{array}{c}
\left.r+n-a_{i j k}\right) \\
n
\end{array}\right)\left[\left(\begin{array}{c}
s+n-b_{i j k} \\
n
\end{array}\right)\right. \\
\left.-\left(\begin{array}{c}
s+n-\min \left\{c_{i}+b_{i j k}-b_{i}, c_{j}+b_{i j k}-b_{j}, c_{k}+b_{i j k}-b_{k}\right\} \\
n
\end{array}\right)\right]
\end{aligned}
$$

and so on.

Thus, for all sufficiently large $(r, s) \in \mathbf{N}^{2}, \operatorname{Card} U_{r s}^{\prime \prime}=\bar{\omega}(r, s)$ where $\bar{\omega}\left(t_{1}, t_{2}\right)$ is the following numerical polynomial:

$$
\bar{\omega}\left(t_{1}, t_{2}\right)=\sum_{i=1}^{d}\left(\begin{array}{c}
t_{1}+n-a_{i} \\
n
\end{array}\right)\left[\left(\begin{array}{c}
t_{2}+n-b_{i} \\
n
\end{array}\right)-\left(\begin{array}{c}
t_{2}+n-c_{i} \\
n
\end{array}\right)\right]
$$




$$
\begin{aligned}
& -\sum_{1 \leqslant i<j \leqslant d}\left(\begin{array}{c}
t_{1}+n-a_{i j} \\
n
\end{array}\right)\left[\left(\begin{array}{c}
t_{2}+n-b_{i j} \\
n
\end{array}\right)\right. \\
& \left.-\left(\begin{array}{c}
t_{2}+n-\min \left\{c_{i}+b_{i j}-b_{i}, c_{j}+b_{i j}-b_{j}\right\} \\
n
\end{array}\right)\right] \\
& +\sum_{1 \leqslant i<j<k \leqslant d}\left(\begin{array}{c}
t_{1}+n-a_{i j k} \\
n
\end{array}\right)\left[\left(\begin{array}{c}
t_{2}+n-b_{i j k} \\
n
\end{array}\right)\right. \\
& \left.-\left(\begin{array}{c}
t_{2}+n-\min \left\{c_{i}+b_{i j k}-b_{i}, c_{j}+b_{i j k}-b_{j}, c_{k}+b_{i j k}-b_{k}\right\} \\
n
\end{array}\right)\right]-\ldots
\end{aligned}
$$

It is clear now that the numerical polynomial $\phi_{M}\left(t_{1}, t_{2}\right)=\omega\left(t_{1}, t_{2}\right)+\bar{\omega}\left(t_{1}, t_{2}\right)$ has all the desired properties.

DEFINITION 5.2: The numerical polynomial $\phi_{M}\left(t_{1}, t_{2}\right)$, whose existence is established by Theorem 5.2, is called a characteristic polynomial of the module $M$ associated with the system of generators $\left\{f_{1}, \ldots, f_{p}\right\}$ (or with the bifiltration $\left(M_{r s}\right)_{r, s \in N}$.)

EXAMPLE 5.2. With the notation of Theorem 5.2, let $n=1$ and let the $A_{1}(K)$ module $M$ be generated by a single element $f$ that satisfies the defining equation $x^{2} f+$ $\partial^{2} f+x \partial f=0$. In other words, $M$ is a factor module of a free $A_{1}(K)$-module $E=A_{1}(K) e$ with a free generator $e$ by its $A_{1}(K)$-submodule $N=A_{1}(K) g$ where $g=\left(x^{2}+\partial^{2}+x \partial\right) e$. By Proposition 4.6, $\{g\}$ is a characteristic set of $N$. Applying Proposition 3.3 (and using the notation of Theorem 5.2), we obtain that $u_{g}=x^{2} e$, $v_{g}=\partial^{2} e$, and

$$
\omega\left(t_{1}, t_{2}\right)=\omega_{\{(2,0)\}}\left(t_{1}, t_{2}\right)=\left(\begin{array}{c}
t_{1}+1 \\
1
\end{array}\right)\left(\begin{array}{c}
t_{2}+1 \\
1
\end{array}\right)-\left(\begin{array}{c}
t_{1}+1-2 \\
1
\end{array}\right)\left(\begin{array}{c}
t_{2}+1 \\
1
\end{array}\right)=2 t_{2}+2 .
$$

Furthermore, formula (5.1) shows that

$$
\bar{\omega}\left(t_{1}, t_{2}\right)=\left(\begin{array}{c}
t_{1}+1-2 \\
1
\end{array}\right)\left[\left(\begin{array}{c}
t_{2}+1 \\
1
\end{array}\right)-\left(\begin{array}{c}
t_{2}+1-2 \\
1
\end{array}\right)\right]=2 t_{1}-2 .
$$

Thus, the characteristic polynomial of the module $M$ associated with the generator $f$ is as follows: $\phi_{M}\left(t_{1}, t_{2}\right)=\omega\left(t_{1}, t_{2}\right)+\bar{\omega}\left(t_{1}, t_{2}\right)=2 t_{1}+2 t_{2}$.

THEOREM 5.3. Let $M$ be a finitely generated $A_{n}(K)$-module and let $\phi_{M}\left(t_{1}, t_{2}\right)=$ $\sum_{i=0}^{n} \sum_{j=0}^{n} a_{i j}\left(\begin{array}{c}t_{1}+i \\ i\end{array}\right)\left(\begin{array}{c}t_{2}+j \\ j\end{array}\right)$ be a characteristic polynomial associated with some finite system of generators $\left\{g_{1}, \ldots, g_{p}\right\}$ of $M$. (We write $\phi_{M}\left(t_{1}, t_{2}\right)$ in the form (3.1) with integer coefficients $a_{i j}, 1 \leqslant i, j \leqslant n$.) Furthermore, let $\Lambda=\left\{(i, j) \in \mathrm{N}^{2} \mid 0 \leqslant i, j \leqslant\right.$ $n$ and $\left.a_{i j} \neq 0\right\}$, and let $\mu=\left(\mu_{1}, \mu_{2}\right)$ and $\nu=\left(\nu_{1}, \nu_{2}\right)$ be the maximal elements of the set $\Lambda$ relative to the lexicographic and reverse lexicographic orders on $\mathbf{N}^{2}$, respectively. 
Then $\mu, \nu$ and the coefficients $a_{n n}, a_{\mu_{1}, \mu_{2}}, a_{\nu_{1}, \nu_{2}}$ of the characteristic polynomial $\phi_{M}\left(t_{1}, t_{2}\right)$ do not depend on the finite system of generators of the $A_{n}(K)$-module $M$ this polynomial is associated with.

Proof: Let $\left\{h_{1}, \ldots, h_{q}\right\}$ be another finite system of generators of the $A_{n}(K)$ module $M$ and let $\left(M_{r s}\right)_{r, s \in Z}$ and $\left(M_{r s}^{\prime}\right)_{r, s \in Z}$ be the the natural bifiltrations associated with the systems of generators $\left\{g_{1}, \ldots, g_{p}\right\}$ and $\left\{h_{1}, \ldots, h_{q}\right\}$, respectively. $\left(M_{r_{s}}=\right.$ $\sum_{i=1}^{p} D_{r s} g_{i}$ and $M_{r s}^{\prime}=\sum_{i=1}^{q} D_{r s} h_{i}$ for $r, s \in \mathrm{N}, M_{r s}=0$ and $M_{r s}^{\prime}=0$, if at least one of the indices $r, s$ is negative.) Furthermore, let $\phi_{M}^{*}\left(t_{1}, t_{2}\right)=\sum_{i=0}^{n} \sum_{j=0}^{n} b_{i j}\left(\begin{array}{c}t_{1}+i \\ i\end{array}\right)\left(\begin{array}{c}t_{2}+j \\ j\end{array}\right)$ $\left(b_{i j} \in \mathbf{Z}\right.$ for $\left.i=0, \ldots, n ; j=0, \ldots, n\right)$ be the characteristic polynomial associated with the system $\left\{h_{1}, \ldots, h_{q}\right\}$, let $\Lambda^{\prime}=\left\{(i, j) \in \mathbf{N}^{2} \mid 0 \leqslant i, j \leqslant n\right.$, and $\left.b_{i j} \neq 0\right\}$, and let $\sigma=\left(\sigma_{1}, \sigma_{2}\right), \varepsilon=\left(\varepsilon_{1}, \varepsilon_{2}\right)$ be the maximal elements of $\Lambda^{\prime}$ relative to the lexicographic and reverse lexicographic orders on $\mathbf{N}^{2}$, respectively. In order to prove the theorem, we should show that $\mu=\sigma, \nu=\varepsilon, a_{n n}=b_{n n}, a_{\mu_{1} \mu_{2}}=b_{\sigma_{1} \sigma_{2}}$, and $a_{\nu_{1} \nu_{2}}=b_{\varepsilon_{1} \varepsilon_{2}}$.

Since $\bigcup_{r, s \in \mathbf{Z}} M_{r s}=\bigcup_{r, s \in \mathbf{Z}} M_{r s}^{\prime}=M$, there exist elements $r_{0}, s_{0} \in \mathbf{N}$ such that $M_{r s} \subseteq M_{r+r_{0}, s+s_{0}}^{\prime}$ and $M_{r s}^{\prime} \subseteq M_{r+r_{0}, s+s_{0}}$ for all $r_{2} s \in \mathbf{N}$. It follows that $\phi_{M}(r, s) \leqslant$ $\phi_{M}^{*}\left(r+r_{0}, s+s_{0}\right)$ and $\phi_{M}^{*}(r, s) \leqslant \phi\left(r+r_{0}, s+s_{0}\right)$ for all sufficiently large $r, s \in \mathbf{N}$, say for all $r \geqslant r_{1}, s \geqslant s_{1}$ where $r_{1}$ and $s_{1}$ are some positive integers. Therefore, $a_{n n}=(n !)^{2} \lim _{r \rightarrow \infty, s \rightarrow \infty}\left(\phi_{M}(r, s) / r^{m} s^{n}\right) \leqslant(n !)^{2} \lim _{r \rightarrow \infty, s \rightarrow \infty}\left(\phi_{M}^{*}\left(r+r_{0}, s+s_{0}\right) / r^{n} s^{n}\right)=$ $(n !)^{2} \lim _{r \rightarrow \infty, s \rightarrow \infty}\left(\phi_{M}^{*}(r, s) / r^{n} s^{n}\right)=b_{n n}$ and similarly $b_{n n} \leqslant a_{n n}$, so that $b_{n n}=a_{n n}$.

If $a_{n n} \neq 0$, then $(n, n) \in \Lambda$ and $(n, n) \in \Lambda^{\prime}$ hence $\mu=\nu=\sigma=\varepsilon=(n, n)$ and $a_{\mu_{1} \mu_{2}}=a_{\nu_{1} \nu_{2}}=b_{\sigma_{1} \sigma_{2}}=b_{\varepsilon_{1} \varepsilon_{2}}=a_{n n}=b_{n n}$. Suppose that $a_{n n}=0$. Then $\left(\mu_{1}, \mu_{2}\right) \neq(n, n), a_{\mu_{1} \mu_{2}} \neq 0$, and the coefficient of the monomial $t_{1}^{\mu_{1}} t_{2}^{\mu_{2}}$ in the polynomial $\phi_{M}\left(t_{1}, t_{2}\right)$ is equal to $\left(a_{\mu_{1} \mu_{2}} / \mu_{1} ! \mu_{2} !\right)$.

Let $s \in \mathbf{N}, s \geqslant s_{1}$, and let $d$ be a positive integer such that $s^{d} \geqslant r_{1}$. By the choice of the elements $\mu$ and $\sigma, \phi_{M}\left(s^{d}, s\right)=\left(a_{\mu_{1} \mu_{2}} / \mu_{1} ! \mu_{2} !\right) s^{d \mu_{1}+\mu_{2}}+o\left(s^{d \mu_{1}+\mu_{2}}\right)$ and $\phi_{M}^{*}\left(s^{d}, s\right)=\left(b_{\sigma_{1} \sigma_{2}} / \sigma_{1} ! \sigma_{2} !\right) s^{d \sigma_{1}+\sigma_{2}}+o\left(s^{d \sigma_{1}+\sigma_{2}}\right)$ for all sufficiently large values of $d$. Since $\phi_{M}\left(s^{d}, s\right) \leqslant \phi^{*}\left(s^{d}+r_{0}, s+s_{0}\right)=\left(b_{\sigma_{1} \sigma_{2}} / \sigma_{1} ! \sigma_{2} !\right) s^{d \sigma_{1}+\sigma_{2}}+o\left(s^{d \sigma_{1}+\sigma_{2}}\right)$ and $\phi_{M}^{*}\left(s^{d}, s\right) \leqslant \phi\left(s^{d}+r_{0}, s+s_{0}\right)=\left(a_{\mu_{1} \mu_{2}} / \mu_{1} ! \mu_{2} !\right) s^{d \mu_{1}+\mu_{2}}+o\left(s^{d \mu_{1}+\mu_{2}}\right)$ for all $s \geqslant s_{1}$, we conclude that $d \mu_{1}+\mu_{2}=d \sigma_{1}+\sigma_{2}$ for all sufficiently large $d \in \mathbf{N}$ and the coefficients of the power $s^{d \mu_{1}+\mu_{2}}$ in the polynomials $\phi_{M}\left(t_{1}, t_{2}\right)$ and $\phi_{M}^{*}\left(t_{1}, t_{2}\right)$ are equal. Therefore, $\mu_{1}=\sigma_{1}, \mu_{2}=\sigma_{2}$ and $a_{\mu_{1} \mu_{2}}=b_{\mu_{1} \mu_{2}}$. The equalities $\nu_{1}=\varepsilon_{1}, \nu_{2}=\varepsilon_{2}$ and $a_{\nu_{1} \nu_{2}}=$ $b_{\nu_{1} \nu_{2}}$ can be proved similarly.

It is clear that if $\left(W_{r}\right)_{r \in Z}$ is the one-dimensional filtration of the Weyl algebra $A_{n}(K)$ introduced in Section 2, then $W_{r} \subseteq D_{r r} \subseteq W_{2 r}$ for all $r \in \mathbf{N}$. Therefore, if $\phi_{M}\left(t_{1}, t_{2}\right)$ and $\psi_{M}(t)$ denote, respectively, our characteristic polynomial and the 
Bernstein polynomial associated with the same finite system of generators of an $A_{n}(M)$ module $M$, then $\psi_{M}(r) \leqslant \phi_{M}(r, r) \leqslant \psi_{M}(2 r)$ for all sufficiently large $r \in \mathbf{Z}$. It follows that $n \leqslant \operatorname{deg} \psi_{M}(t)=\operatorname{deg} \phi_{M}\left(t_{1}, t_{2}\right) \leqslant 2 n$ and $M \in \mathcal{B}_{n}$ if and only if $\operatorname{deg} \phi_{M}\left(t_{1}, t_{2}\right)=$ $n$.

The following example shows that a characteristic polynomial $\phi_{M}\left(t_{1}, t_{2}\right)$ of a finitely generated $A_{n}(K)$-module $M$ can carry more invariants (that is, numbers that do not depend on the choice of a system of generators the characteristic polynomial is associated with) than the Bernstein polynomial $\psi_{M}(t)$.

EXAMPLE 5.3. With the notation of Theorem 5.2, let the $A_{1}(K)$-module $M$ be generated by a single element $f$ that satisfies the defining equation

$$
x^{a} \partial^{b} f+\partial^{a+b} f=0
$$

where $a$ and $b$ are some positive integers. In other words, $M$ is a factor module of a free $A_{1}(K)$-module $E=A_{1}(K) e$ with a free generator $e$ by its $A_{1}(K)$-submodule $N=A_{1}(K) g$ where $g=\left(x^{a} \partial^{b}+\partial^{a+b}\right) e$. By Proposition 4.6, $\{g\}$ is a characteristic set of the module $N$. Since $u_{g}=x^{a} \partial^{b} e$ and $v_{g}=\partial^{a+b} e$, we obtain (using the notation of Theorem 5.2) that $\omega\left(t_{1}, t_{2}\right)=\omega_{\{(a, b)\}}\left(t_{1}, t_{2}\right)=\left(\begin{array}{c}t_{1}+1 \\ 1\end{array}\right)\left(\begin{array}{c}t_{2}+1 \\ 1\end{array}\right)-$ $\left(\begin{array}{c}t_{1}+1-a \\ 1\end{array}\right)\left(\begin{array}{c}t_{2}+1-b \\ 1\end{array}\right)=b t_{1}+a t_{2}+a+b-a b$. Furthermore, formula (5.1) shows that $\bar{\omega}\left(t_{1}, t_{2}\right)=\left(\begin{array}{c}t_{1}+1-a \\ 1\end{array}\right)\left[\left(\begin{array}{c}t_{2}+1-b \\ 1\end{array}\right)-\left(\begin{array}{c}t_{2}+1-(a+b) \\ 1\end{array}\right)\right]=a t_{1}+a(1-a)$. Thus, the characteristic polynomial of the module $M$ associated with the generator $f$ is as follows: $\phi_{M}\left(t_{1}, t_{2}\right)=\omega\left(t_{1}, t_{2}\right)+\bar{\omega}\left(t_{1}, t_{2}\right)=(a+b) t_{1}+a t_{2}+2 a+b-a b-a^{2}$.

The Bernstein polynomial $\psi_{M}(t)$ associated with the generator $f$ of the $A_{1}(K)$ module $M$ can be obtained from the exact sequence of finitely generated filtered modules

$$
0 \longrightarrow F^{a+b} \stackrel{\alpha}{\longrightarrow} E \stackrel{\pi}{\longrightarrow} M \longrightarrow 0
$$

where $M$ and $E$ are equipped, respectively, with the filtrations $\left(W_{r} e\right)_{r \in \mathbf{Z}}$ and $\left(W_{r} f\right)_{r \in \mathbf{Z}}$ defined in Section 2 , and $F^{a+b}$ is a free filtered $A_{1}(K)$-module with a single free generator $h$ and filtration $\left(W_{r-(a+b)} h\right)_{r \in \mathbf{Z}}$. (Here $\pi$ denotes the natural $A_{1}(K)$-epimorphism of $E$ onto $M$ that maps $e$ onto $f$, and $\alpha$ is the natural $A_{1}(K)$-epimorphism of the free filtered module $F^{a+b}$ onto the $A_{1}(K)$-module $N \subseteq M$ equipped with the filtration $\left.\left(W_{r} g\right)_{r \in \mathbf{Z}}, \alpha(h)=g.\right)$

Since $\operatorname{dim}_{K} W_{r}=\operatorname{Card}\left\{x^{i} \partial^{j} \mid i+j \leqslant r\right\}=\left(\begin{array}{c}r+2 \\ 2\end{array}\right)$ for all $r \in \mathbf{N}, \operatorname{dim}_{K}\left(W_{r-(a+b)} h\right)$ $=\left(\begin{array}{c}r+2-(a+b) \\ 2\end{array}\right)$ for all sufficiently large $r \in \mathbf{Z}$ whence $\psi_{M}(r)=\operatorname{dim}_{K}\left(W_{r} e\right)-$ 
$\operatorname{dim}_{K}\left(W_{r-(a+b)} h\right)=\left(\begin{array}{c}r+2 \\ 2\end{array}\right)-\left(\begin{array}{c}r+2-(a+b) \\ 2\end{array}\right)$ for all sufficiently large $r \in \mathbf{Z}$. Therefore, $\psi_{M}(t)=\left(\begin{array}{c}t+2 \\ 2\end{array}\right)-\left(\begin{array}{c}t+2-(a+b) \\ 2\end{array}\right)=(a+b) t-((a+b)(a+b-3) / 2)$.

Comparing the polynomials $\psi_{M}(t)$ and $\phi_{M}\left(t_{1}, t_{2}\right)$ we see that the first polynomial carries two invariants, its degree 1 and the leading coefficient $a+b$, while $\phi_{M}\left(t_{1}, t_{2}\right)$ carries three such invariants, its total degree $1, a+b$, and $a$. Thus, $\phi_{M}\left(t_{1}, t_{2}\right)$ gives both parameters $a$ and $b$ of the equation (5.2) while the Bernstein polynomial $\psi_{M}(t)$ gives just the sum of the parameters.

\section{REFERENCES}

[1] T. Becker and V. Weispfenning, Gröbner bases. A computational approach to commutative algebra (Springer-Verlag, Berlin, Heidelberg, New York, 1993).

[2] I.N. Bernstein, 'Modules over the ring of differential operators. A study of the fundamental solutions of equations with constant coefficients', Functional Anal. Appl. 5 (1971), 89-101.

[3] I.N. Bernstein, 'The analytic continuation of generalized functions with respect to a parameter', Functional Anal. Appl. 6 (1972), 273-285.

[4] J.- E. Björk, Rings of differential operators (North Holland Publishing Company, Amsterdam, New York, 1979).

[5] P.J. Cameron, Combinatorics. Topics, techniques, algorithms (Cambridge University Press, Cambridge, 1994).

[6] D. Eisenbud, Commutative algebra with a view toward algebraic geometry (Springer-Verlag, Berlin, Heidelberg, New York, 1995).

[7] M. Insa and F.Pauer, 'Gröbner bases in rings of differential operators', in Gröbner Bases and Applications (Cambridge Univ. Press, New York, 1998), pp. 367-380.

[8] E.R. Kolchin, Differential algebra and algebraic groups (Academic Press, New York, 1973).

[9] M.V. Kondrateva, A.B. Levin, A.V. Mikhalev and E. V. Pankratev, 'Computation of dimension polynomials', Internat. J. Algebra Comput. 2 (1992), 117-137.

[10] M.V. Kondrateva, A.B. Levin, A.V. Mikhalev and E.V. Pankratev, Differential and difference dimension polynomials (Kluwer Academic Publishers, Dordrecht, Boston, London, 1999).

Department of Mathematics

The Catholic University of America

Washington DC 20064

United States of America

e-mail: levin@cua.edu 